\title{
Distribución y ajuste del comportamiento bajo condiciones de entrega de agua contingente y no contingente
}

\author{
Distribution and Adjustment of Behavior under Contingent and Mon-contingent \\ Conditions of Water Delivery \\ Distribuição e ajuste do comportamento sob condições de entrega de água \\ contingente e não contingente
}

\author{
Mario Serrano, Darcy Martínez, Édgar Montes* \\ Universidad Veracruzana, México
}

Doi: dx.doi.org/10.12804/apl33.02.2015.11

\section{Resumen}

La observación de relaciones funcionales similares bajo contingencias pavlovianas y operantes podría apoyar la idea de que los comportamientos respondiente y operante son el resultado de un mismo proceso. En el presente estudio se evaluaron los efectos de la secuencia de exposición a la entrega de agua no contingente y contingente sobre la ocurrencia, distribución temporal y ajuste del comportamiento. Entre sujetos se utilizaron luces o tonos como estímulos antecedentes. Las respuestas requeridas bajo cada condición de entrega de agua (asomarse y apretar la palanca) aumentaron y disminuyeron en correspondencia con el tipo de contingencia vigente. No se observaron diferencias en la distribución temporal de ambas respuestas cuando fueron requeridas por la contingencia en vigor. En ambas secuencias de exposición se observó que el ajuste conductual fue más elevado en la segunda condición de entrega de agua que en la primera. Los resultados se discuten atendiendo a la posibilidad de un mismo proceso conductual bajo ambas condiciones de entrega de agua.

Palabras clave: condicionamiento respondiente; condicionamiento operante; estímulos visuales versus auditivos; intervalo fijo; apretar la palanca; ratas.

\section{Abstract}

The observation of similar functional relations under Pavlovian and operant contingencies could support the idea that both respondent and operant behaviors are the result of the same process. The effects of the sequence of exposure to non-contingent and contingent water delivery on the occurrence, temporal distribution, and

* Mario Serrano, Centro de Estudios e Investigaciones en Conocimiento y Aprendizaje Humano, Universidad Veracruzana, México; Darcy Martínez, Centro de Estudios e Investigaciones en Conocimiento y Aprendizaje Humano, Universidad Veracruzana, México; Édgar Montes, Centro de Estudios e Investigaciones en Conocimiento y Aprendizaje Humano Universidad Veracruzana, México. La presente investigación fue posible gracias al Proyecto 180619, del Consejo Nacional de Ciencia y Tecnología (México), a cargo del primer autor. Los autores agradecen las observaciones y sugerencias de los revisores anónimos para mejorar el manuscrito. La correspondencia relacionado con este artículo se debe dirigir a Mario Serrano, Universidad Veracruzana, Centro de Estudios e Investigaciones en Conocimiento y Aprendizaje Humano. Correo electrónico: mserrano@uv.mx

Para citar este artículo: Serrano, M., Martínez, D. \& Montes, É. (2015). Distribución y ajuste del comportamiento bajo condiciones de entrega de agua contingente y no contingente. Avances en Psicología Latinoamericana, 33(2), 333-345. doi: dx.doi. org/10.12804/ap133.02.2015.11 
behavioral adjustment were assessed. Lights or tones were used as antecedent stimuli between subjects. The required responses under each condition of water delivery (nose-poking and lever-pressing) increased and decreased in correspondence with the ongoing contingency. No differences in the temporal distribution of both responses were observed when they were required by the ongoing contingency. Under both sequences of exposure the behavioral adjustment was higher in the second than in the first condition of water delivery. The results are discussed considering the possibility of a single behavioral process under both conditions of water delivery.

Key words: respondent conditioning; operant conditioning; visual versus auditory stimuli; fixed-interval; lever-pressing; rats.

\section{Resumo}

A observação de relações funcionais similares sob contingencias Pavlovianas e operantes poderia apoiar a ideia de que os comportamentos respondente e operante são o resultado de um mesmo processo. No presente estudo se avaliaram os efeitos da sequência de exposição à entrega de água não contingente e contingente sobre a ocorrência, distribuição temporal e ajuste do comportamento. Entre sujeitos utilizaram-se luzes ou tonos como estímulos antecedentes. As respostas requeridas sob cada condição de entrega de água (assomar-se e puxar a alavanca) aumentaram e diminuíram em correspondência com o tipo de contingência vigente. Não se observaram diferenças na distribuição temporal de ambas as respostas quando foram requeridas pela contingência em vigor. Em ambas as sequencias de exposição observou-se que o ajuste comportamental foi mais elevado na segunda condição de entrega de água que na primeira. Os resultados se discutem atendendo à possibilidade de um mesmo processo comportamental sob ambas as condições de entrega de água.

Palavras chave: condicionamento respondente; condicionamento operante; estímulos visuais versus auditivos; intervalo fixo; puxar a alavanca; ratos.
En contraste con la conducta respondiente, la operante es espontánea en el sentido de que cuando se observa que ocurre no se puede identificar un estímulo antecedente que la provoque. El carácter provocado versus el espontáneo de cada tipo de comportamiento derivó en procedimientos de condicionamiento también distintos en cada caso. En el condicionamiento respondiente el estímulo reforzante debe ser contingente a otro estímulo ( $e$. g. Pavlov, 1927); mientras que en el condicionamiento operante el estímulo reforzante debe ser contingente a una respuesta (e. g. Skinner, 1938). Diferentes estudios, sin embargo, han mostrado que entregar el estímulo reforzante sin referencia a una respuesta predefinida experimentalmente incrementa la frecuencia de las actividades espontáneas que lo preceden (e. g. Skinner, 1948), así como hacer contingente un estímulo reforzante sobre una respuesta (e.g. Shapiro, 1961) o sobre un estímulo (e. g. Brown \& Jenkins, 1968) fortalece respondientes y operantes prototípicas, respectivamente.

De acuerdo con Pear y Eldridge (1984), la existencia de dos operaciones de reforzamiento ha sido razón suficiente para mantener la dicotomía respondiente-operante para muchos analistas experimentales de la conducta. Tales autores han sugerido que para demostrar que las conductas respondiente y operante condicionadas constituyen o no manifestaciones de un mismo proceso subyacente, es necesario demostrar o descartar un isomorfismo del comportamiento bajo ambos tipos de procedimiento en niveles de análisis tanto topográficos como funcionales. El nivel topográfico de análisis se refiere a la posibilidad de identificar similitudes o diferencias de "grano fino" entre el comportamiento controlado por procedimientos respondientes y operantes. Que el uso de agua versus comida como estímulo reforzante produzca las mismas diferencias en la morfología de la respuesta de picar la tecla por palomas bajo procedimientos respondientes (e.g. Jenkins \& Moore, 1973) y bajo procedimientos operantes (e.g. Wollin, 1968) es un ejemplo del nivel topográfico de análisis. 
El nivel funcional de análisis, por otro lado, se refiere ya sea a la identificación de efectos diferentes sobre una misma respuesta al manipular cada tipo de contingencia, o bien a la observación de efectos similares o diferentes por parte de una misma manipulación experimental bajo ambos tipos de procedimiento. En el primer caso, una estrategia experimental pertinente consistiría en manipular la duración de la señal que precede al choque eléctrico y la del intervalo respuesta-reforzador en un procedimiento de supresión condicionada (véase Estes \& Skinner, 1942). En el segundo, una posibilidad radica en comparar los gradientes de generalización que se obtienen después de un entrenamiento de discriminación bajo condiciones paramétricamente equivalentes de reforzamiento no contingente (NC) versus reforzamiento contingente (C) (e.g. Weiss \& Weissman, 1992).

El presente estudio es un intento inicial por identificar similitudes y diferencias al nivel funcional de análisis entre el comportamiento controlado por procedimientos respondientes y procedimientos operantes paramétricamente equivalentes. Específicamente, el experimento que aquí se reporta evaluó los efectos de la secuencia de exposición a las condiciones de entrega de agua $\mathrm{NC}$ y $\mathrm{C}$ sobre la ocurrencia, distribución temporal y ajuste del comportamiento. Estudios previos han comparado ambas condiciones de reforzamiento utilizando programas múltiples con dos componentes de intervalo fijo (IF), o bien dos componentes de intervalo variable, uno de los cuales eventualmente fue sustituido por un componente temporalmente análogo, pero que entregó el reforzamiento al margen de las respuestas del organismo, es decir, por un programa de tiempo fijo o bien de tiempo variable, respectivamente (e. g. Appel \& Hiss, 1962; Lattal \& Maxy, 1971; Zeiler, 1968). En general, en estos estudios se observó que la tasa de una respuesta instrumental fue menor durante los componentes de reforzamiento $\mathrm{NC}$ que durante los componentes de reforzamiento $\mathrm{C}$.
Una nueva comparación entre condiciones de reforzamiento $\mathrm{NC}$ y C parece necesaria por, al menos, una razón, simple pero importante. En los estudios arriba citados los efectos del reforzamiento NC se determinaron sobre la base de los decrementos en la respuesta requerida bajo la condición de reforzamiento $\mathrm{C}$ (i. e. picar una tecla o apretar una palanca), más que a partir de segmentos conductuales que emergieron puramente a partir de contingencias entre estímulos. Sobre el particular, estudios más recientes sugieren que las respuestas que emergen bajo condiciones de reforzamiento NC pueden tener un efecto facilitador más que incompatible sobre respuestas procuradoras de reforzamiento, tal como los meros decrementos en la respuesta requerida bajo condiciones de reforzamiento $\mathrm{C}$ podrían sugerir (e.g. López \& Menez, 2012). En línea con las similitudes funcionales entre contingencias pavlovianas y operantes descritas al inicio del presente escrito, es posible que manipular la secuencia de exposición a las condiciones de entrega de agua $\mathrm{NC}$ y $\mathrm{C}$ revele que el efecto facilitador recién mencionado es independiente del tipo de relación de contingencia enfrentada inicialmente por el organismo.

\section{Método}

\section{Sujetos}

Se utilizaron cuatro ratas Wistar macho, experimentalmente ingenuas y de aproximadamente tres meses de edad al inicio del experimento. Siete días antes de iniciar el experimento y a lo largo de este último todas las ratas fueron sometidas a un régimen de privación de agua de $23 \mathrm{~h}$ diarias con acceso libre al alimento (Purina Rodent Lab Chow 5001®). Después de cada sesión experimental las cuatro ratas recibieron $30 \mathrm{~min}$ de acceso libre al agua en sus jaulas hogar, las cuales se ubicaron en una colonia con temperatura controlada y un ciclo luz/oscuridad 12:12. 


\section{Aparatos}

Se utilizaron cuatro cámaras de condicionamiento operante (ENV-008) manufacturadas por la empresa Med Associaties Inc. ${ }^{\circledR}$. Las paredes anteriores y posteriores de las cámaras eran de aluminio y las paredes laterales eran de acrílico transparente. En el centro de la pared anterior de cada cámara, a $2 \mathrm{~cm}$ del piso de rejilla, se colocó una apertura para un dispensador de agua (ENV202M-S) provisto con un detector de entradas (ENV-254). El dispensador proporcionó $.01 \mathrm{~cm}^{3}$ de agua en cada activación. A $6 \mathrm{~cm}$ del piso de rejilla y a $.5 \mathrm{~cm}$ de las paredes laterales izquierda y derecha, en cada caja se colocaron dos palancas (ENV-112CM) que requirieron una fuerza de $.25 \mathrm{~N}$ para ser operadas. A $14 \mathrm{~cm}$ del piso de rejilla, arriba de cada palanca, se colocó un módulo de estímulo triple (ENV-222M) que se iluminó con un led de color rojo. A $17 \mathrm{~cm}$ del piso de rejilla se colocó un sonalert que emitió un tono de $2900 \mathrm{~Hz}$ en cada activación. El sonalert (ENV-223AM) se colocó en la esquina superior izquierda de la pared anterior de las cámaras. Las cámaras se colocaron dentro de cubículos de aislamiento acústico (ENV022MD-27), provistos cada uno con un ventilador que facilitó la circulación del aire y proporcionó ruido blanco constante. Para la programación y registro de los eventos experimentales se utilizó una computadora de escritorio estándar (HP Compac Pro 6305), equipada con una interfaz (SG-6510DA) y software (SOF-735) especializados.

\section{Procedimiento}

Las cuatro ratas fueron expuestas directamente a la entrega de agua $\mathrm{NC}$ o $\mathrm{C}$, de acuerdo con programas definidos temporalmente (Schoenfeld \& Cole, 1972) paramétricamente equivalentes. El ciclo $\mathrm{T}$ tuvo una duración de $30 \mathrm{~s}$ y se dividió en subciclos $\mathrm{t}^{\mathrm{D}} \mathrm{y} \mathrm{t}^{\Delta}$ con una duración de $15 \mathrm{~s}$ cada uno. El subciclo $\mathrm{t}^{\mathrm{D}}$ se correlacionó con los leds rojos encima de cada palanca (ratas 1 y 3 ) o bien con el tono de $2900 \mathrm{~Hz}$ (ratas 2 y 4). El subciclo t ${ }^{\Delta}$ no se correlacionó con estímulo exteroceptivo alguno y las respuestas emitidas durante dicho subciclo nunca tuvieron consecuencias. Bajo la condición de entrega de agua $\mathrm{NC}$, el dispensador de agua se activó al final del subciclo $\mathrm{t}^{\mathrm{D}}$ independientemente de la actividad de las ratas. Bajo la condición de entrega de agua $C$, la primera respuesta en el interior del subciclo $\mathrm{t}^{\mathrm{D}}$ sobre cualquiera de las dos palancas disponibles activó el dispensador de agua y canceló la señal exteroceptiva correlacionada con dicho subciclo. En todos los casos se dejó transcurrir el remanente temporal del subciclo $\mathrm{t}^{\mathrm{D}}$ antes de iniciar el subciclo $\mathrm{t}^{\Delta}$.

Las ratas 1 y 2 fueron expuestas a la secuencia NC-C; mientras que las ratas 3 y 4 fueron expuestas a la secuencia C-NC. En ambas condiciones se midieron la duración de las respuestas de asomarse al bebedero y el número de respuestas de apretar las palancas. Se utilizó la duración y no la frecuencia de las respuestas de asomarse al bebedero debido a que estudios previos han sugerido que la primera es más sensible a las manipulaciones experimentales implementadas que la segunda (Holland, 2000). Cada condición de entrega de agua estuvo vigente durante 30 sesiones, consistentes en 60 ciclos $\mathrm{T}$, las cuales se condujeron de lunes a viernes siempre en el mismo orden y horario. Los días en los que no se condujeron sesiones experimentales las ratas recibieron $60 \mathrm{~min}$ de acceso al agua en sus jaulas hogar, en el mismo horario en el que tuvieron acceso en los días de experimentación.

\section{Resultados}

La figura 1 muestra la proporción promedio del tiempo de las respuestas de asomarse al bebedero y de las respuestas de apretar las palancas en las primeras y las últimas cinco sesiones de cada condición de entrega de agua. El total del tiempo de las respuestas de asomarse al bebedero y de apretar las palancas se basó en la sumatoria de cada variable en cada una de las condiciones de entrega de agua 


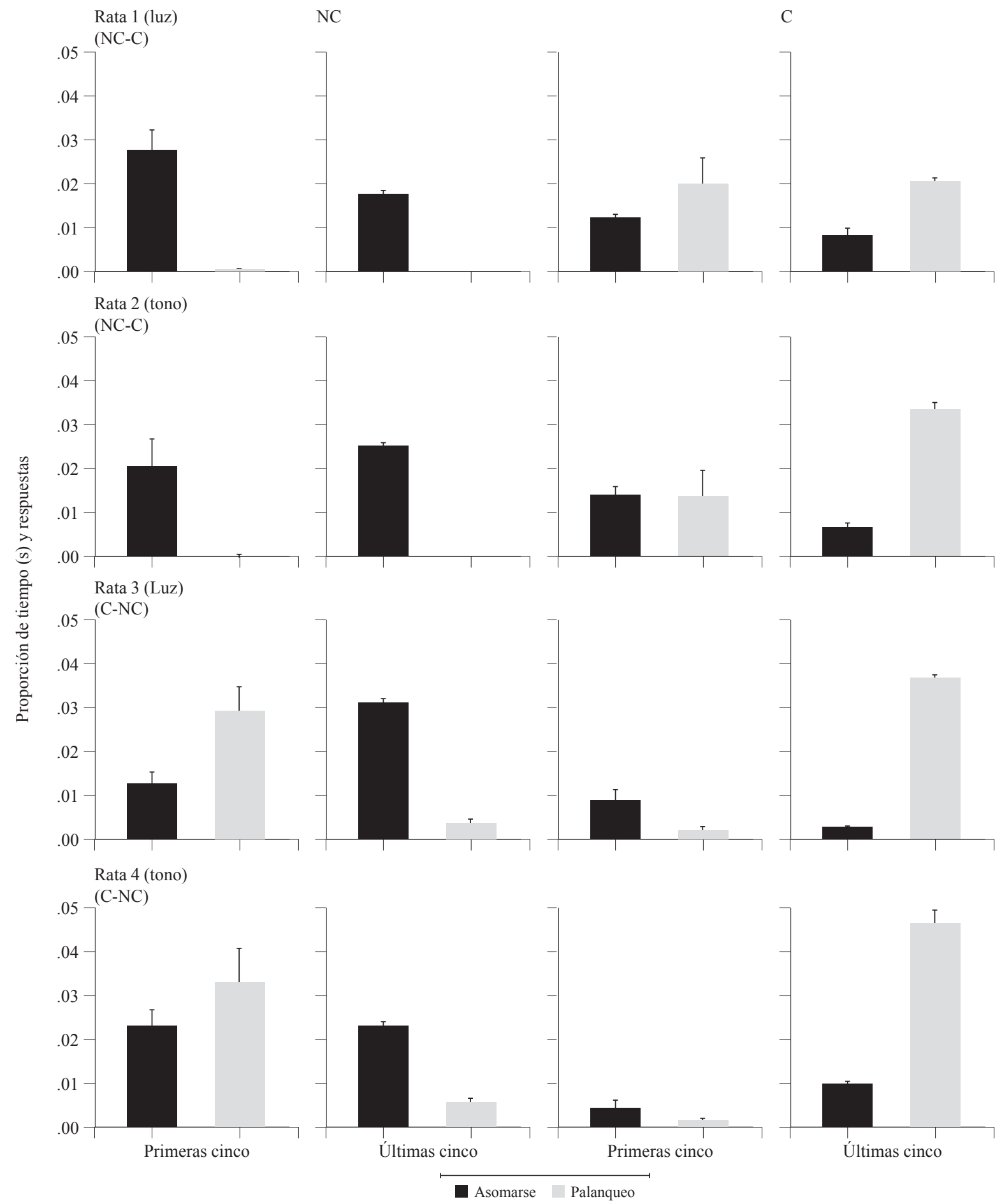

Figura 1. Proporción promedio del tiempo de las respuestas de asomarse al bebedero (barras negras) y de las respuestas de apretar las palancas (barras blancas) en las primeras (primera y tercera columnas) y las últimas (segunda y cuarta columnas) cinco sesiones de las condiciones de entrega de agua NC (columnas de la izquierda) y C (columnas de la derecha). Las barras de error representan el error estándar de la media 
implementadas. En la figura se observa que para todas las ratas la proporción del tiempo de las respuestas de asomarse al bebedero es mayor que la proporción de respuestas de apretar las palancas en las primeras cinco sesiones de la condición inicial de entrega de agua a las que fueron expuestas. En las primeras cinco sesiones de la segunda condición de entrega de agua a las que fueron expuestas, para las ratas 1, 3 y 4 se observa que la proporción de respuestas de apretar las palancas es mayor que la proporción del tiempo de las respuestas de asomarse al bebedero. Para la rata 2, la proporción de respuestas de apretar las palancas y la proporción del tiempo de las respuestas de asomarse al bebedero fueron prácticamente equivalentes. En las últimas cinco sesiones de la condición de entrega de agua $\mathrm{NC}$, para todas las ratas se observa que la proporción del tiempo de las respuestas de asomarse al bebedero es superior a la proporción de respuestas de apretar las palancas. En las últimas cinco sesiones de la condición de entrega de agua $\mathrm{C}$, la proporción de respuestas de apretar las palancas es mayor que la proporción del tiempo de las respuestas de asomarse al bebedero para todas las ratas. En general, en la figura 1 se observa que en el caso de las respuestas requeridas en cada condición de entrega de agua, las ratas expuestas a los tonos mostraron proporciones finales de tiempo y de respuestas más elevadas que las ratas expuestas a las luces, excepto en el caso de las ratas 3 y 4 , en la condición de entrega de agua NC.

La figura 2 muestra el patrón de ejecución dentro del intervalo entre entregas de agua de la proporción de tiempo de las respuestas de asomarse al bebedero y de las respuestas de apretar las palancas, promedio de las últimas cinco sesiones experimentales en cada condición de entrega de agua. El total del tiempo de las respuestas de asomarse al bebedero y de las respuestas de apretar las palancas se basó en la sumatoria de cada variable entre los diferentes subintervalos de $1.5 \mathrm{~s}$ del ciclo T. La proporción del tiempo de las respuestas de asomarse al bebedero está desplegada del inicio del subciclo $\mathrm{t}^{\mathrm{D}}$ al final del subciclo $\mathrm{t}^{\Delta}$, mientras la proporción de las respuestas de apretar las palancas está desplegada del inicio del subciclo $\mathrm{t}^{\Delta}$ al final del subciclo $\mathrm{t}^{\mathrm{D}}$. Esta diferencia en el despliegue de los datos se consideró necesaria para identificar similitudes o diferencias en las "carreras" de ambas variables dependientes. En la figura 2 se observa que, independientemente de la secuencia de exposición, para la mayoría de las ratas la respuesta requerida en la condición inicial de entrega de agua a la que fueron expuestas incrementó de forma positivamente acelerada; mientras que la respuesta requerida en la segunda condición incrementó de forma negativamente acelerada. Una excepción parcial fue la rata 2 , en el caso de la proporción de tiempo de las respuestas de asomarse al bebedero, requerida en la primera condición de entrega de agua a la que fue expuesta. En este caso, la variable incrementó inicialmente de manera positivamente acelerada y posteriormente lo hizo de manera negativamente acelerada.

En algunos estudios conducidos en el marco de la psicología interconductual (véase Ribes \& López, 1985), el comportamiento bajo diferentes condiciones de entrega de agua se ha estimado a partir de índices de ajuste de los que, en términos aristotélicos, se asume describen cuantitativamente la actualización de lo que está en potencia en la situación (e.g. Serrano, Castellanos, Cortés-Zúñiga, De la Sancha-Villa \& Guzmán-Díaz, 2011). Así, por ejemplo, bajo la entrega de agua $\mathrm{NC}$ el ajuste se ha estimado en términos de la razón del tiempo de asomarse al bebedero en presencia del agua y el tiempo de disponibilidad de ésta, restando a dicha razón la proporción del tiempo de asomarse al bebedero en ausencia del agua respecto del tiempo total de la sesión. Bajo la entrega de agua $\mathrm{C}$ el ajuste se ha estimado a partir de la razón entre las entregas de agua producidas y las programadas, multiplicándola por la proporción de respuestas por reforzador (véase Serrano, 2009). La figura 3 muestra ambos índices de ajuste en las condiciones de entrega de agua $\mathrm{NC}$ y $\mathrm{C}$, promedio de las últimas cinco sesiones experimentales en cada 


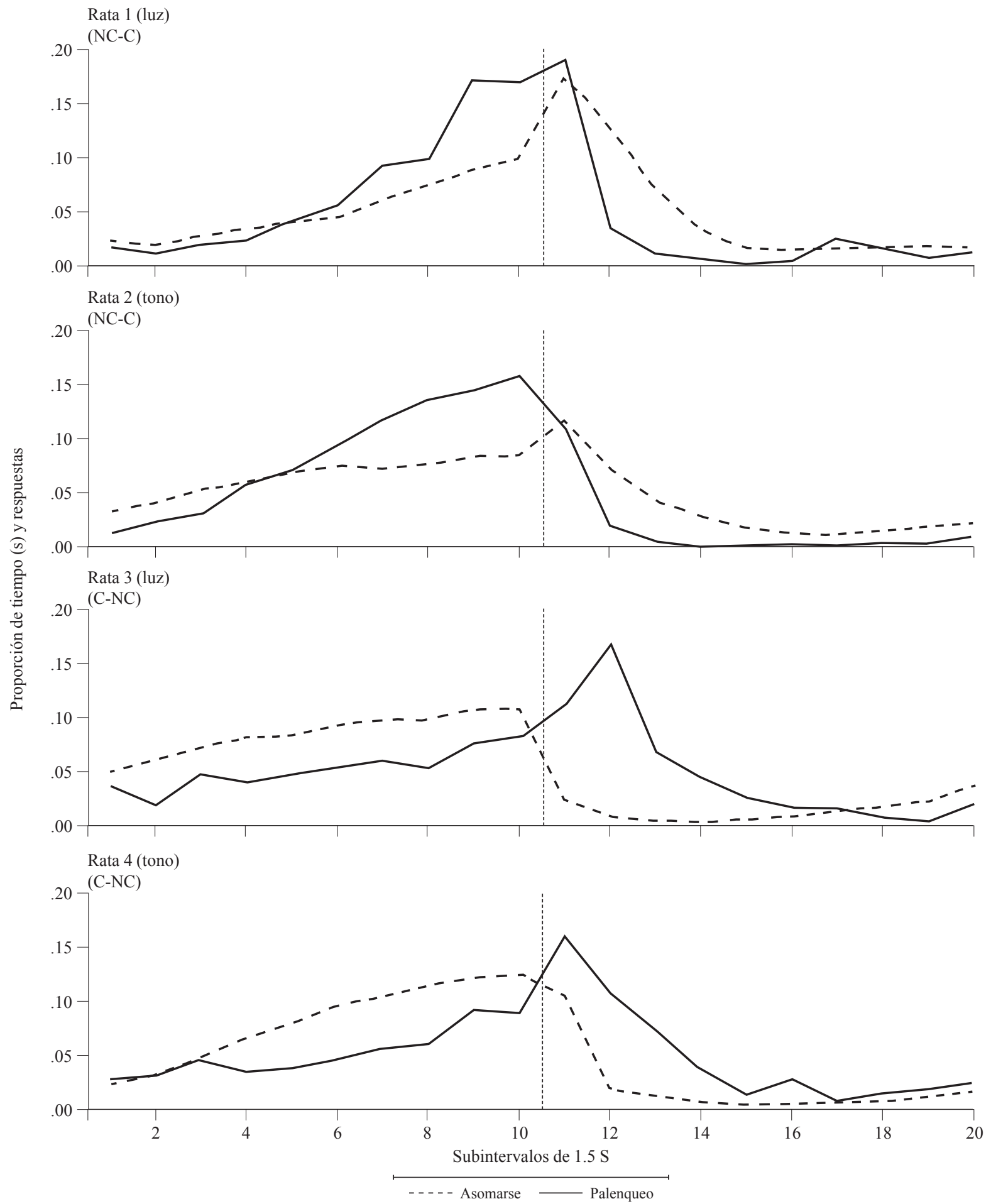

Figura 2. Proporción de tiempo de las respuestas de asomarse al bebedero (línea discontinua) en la condición de entrega de agua $\mathrm{NC}$ y de las respuestas de apretar las palancas (línea continua) en la condición de entrega de agua C dentro del intervalo entre entregas de agua, promedio de las últimas cinco sesiones experimentales en cada condición 

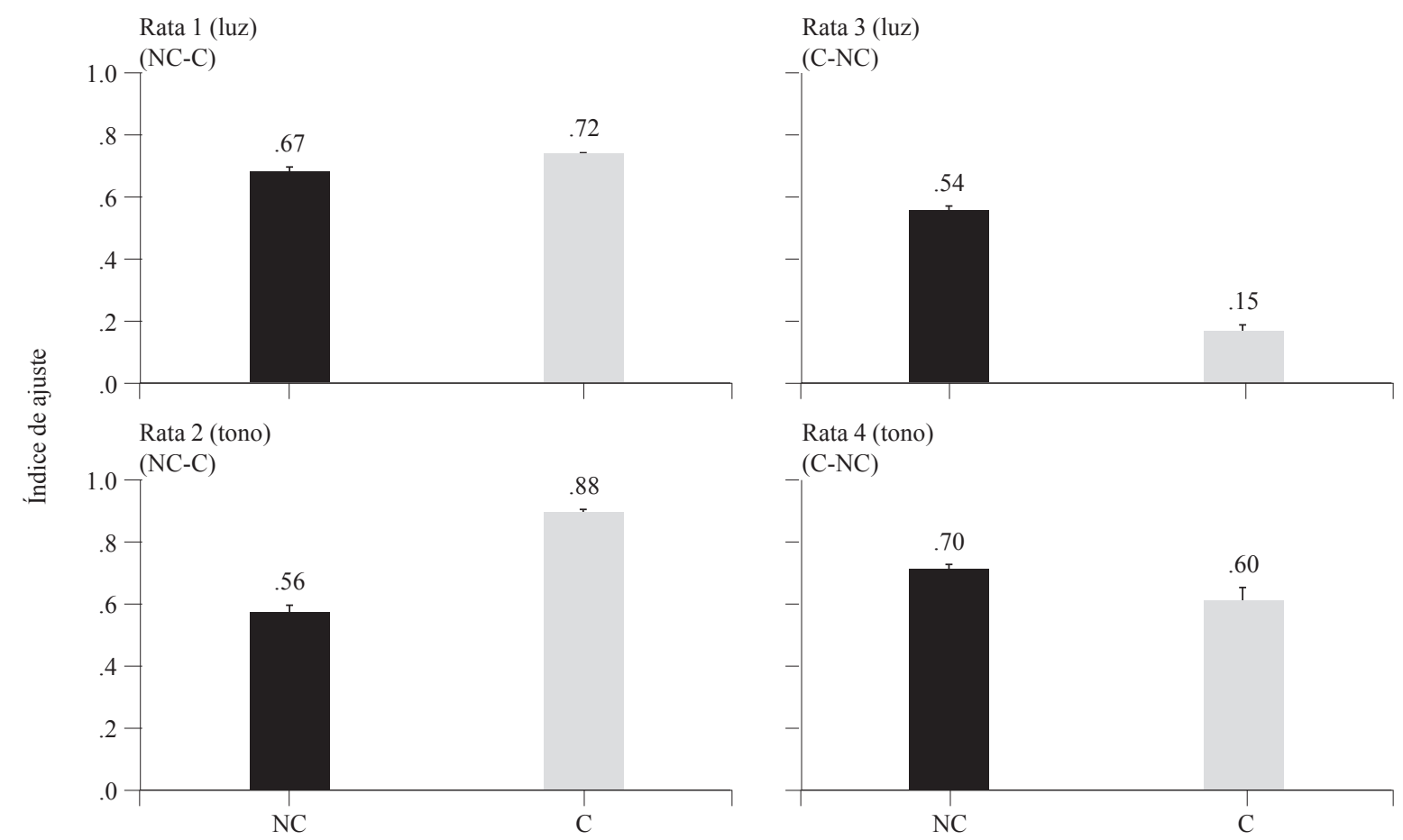

Figura 3. Índices de ajuste para cada rata, promedio de las últimas cinco sesiones experimentales en cada condición de entrega de agua. Las barras de error representan el error estándar de la media

condición. La figura muestra que para todas las ratas los índices de ajuste fueron superiores en la segunda condición de entrega de agua a la que fueron expuestas que los índices de ajuste en la primera condición. En la figura también se observa que el efecto fue mayor para las ratas 1 y 2 en la condición C (índices de ajuste promedio de $.72 \mathrm{y}$ .88 , respectivamente) que para las ratas 3 y 4 en la condición NC (índices de ajuste promedio de .54 у .70 , respectivamente).

\section{Discusión}

El presente experimentó evaluó los efectos de la secuencia de exposición a las condiciones de entrega de agua NC y C sobre la ocurrencia, distribución temporal y ajuste del comportamiento. Se observó que las respuestas requeridas por cada tipo de arreglo contingencial predominaron en cada condición de entrega de agua. Dicho en otros términos, la respuesta de asomarse al bebedero prevaleció bajo la entrega de agua $\mathrm{NC}$, mientras las respuestas de apretar las palancas lo hicieron bajo la condición de entrega de agua C. Sobre la distribución de ambas respuestas en el interior del intervalo entre entregas de agua se observó que, independientemente de la secuencia de exposición, la carrera de la respuesta requerida en la condición inicial de entrega de agua incrementó de manera positivamente acelerada; entre tanto, la carrera de la respuesta requerida en la segunda condición lo hizo de forma negativamente acelerada. También al margen de la secuencia de exposición se observó que el ajuste del comportamiento fue mayor $-\mathrm{O}$ cuando menos ligeramente mayor - en la segunda condición de entrega de agua que en la primera; principalmente en el caso de la secuencia NC-C en la segunda de ambas condiciones. El único efecto observado por el tipo de señal utilizada tuvo lugar en la frecuencia de repuestas de apretar la palanca, siendo mayor en el caso de las señales auditivas que en el caso de las señales visuales. 
Sobre los resultados del presente estudio se pueden hacer varios comentarios de potencial importancia para diversas áreas de investigación en el análisis experimental de la conducta. En el caso de la distinción entre conducta respondiente y conducta operante, como se señaló al principio del presente escrito, estudios clásicos mostraron que el tránsito del reforzamiento $\mathrm{C}$ al NC produce un decremento en la respuesta preespecificada por el experimentador (e. g. Appel \& Hiss, 1962; Lattal \& Maxy, 1971; Zeiler, 1968). Como era de esperarse, los resultados del presente estudio reprodujeron los efectos relativos a la disminución de la respuesta de apretar la palanca al cambiar de una condición en la que el reforzamiento depende de las respuestas del organismo a una en la que el reforzamiento ocurre al margen de su actividad. En los citados estudios, igualmente, se señaló que dicho cambio en las condiciones de reforzamiento prácticamente no afectaba la distribución temporal del responder en el interior del intervalo entre reforzadores. Aunque en el presente estudio las respuestas de apretar las palancas no se analizaron hacia el final de las condiciones de entrega de agua $\mathrm{NC}$, se observó que la distribución temporal de la respuesta requerida en dicha condición siguió un patrón parcialmente inverso al observado para la respuesta instrumental original.

La relevancia de que el patrón de tiempo de asomarse al bebedero en la condición de entrega de agua NC se haya incrementado de una manera negativamente acelerada dentro del ciclo $\mathrm{T}$ para las ratas 3 y 4 depende del ámbito de investigación particular que se tome como marco de referencia. Skinner (1948), por ejemplo, informó que las respuestas bajo la entrega de alimento NC seguían un patrón de festón similar al que se observa bajo un programa de IF en el que la presentación del alimento depende de las respuestas discretas emitidas sobre un operando. Que su registro se haya basado en los cierres de un microinterruptor y en el presente estudio se haya utilizado la duración de la respuesta de asomarse al bebedero en sí misma, en realidad no parece ofrecer una explicación en torno de las diferencias entre los patrones dentro del intervalo entre reforzadores de ambos estudios. Las respuestas de palanqueo para las ratas expuestas a la secuencia $\mathrm{NC}-\mathrm{C}$, principalmente en el caso de la rata 2 , también incrementaron de una manera negativamente acelerada en la condición C; mientras el tiempo de la respuesta de asomarse al bebedero lo hizo de una manera positivamente acelerada en la condición NC, es decir, de manera análoga a las respuestas de apretar las palancas para las ratas 3 y 4 en la condición de entrega de agua $\mathrm{C}$.

Por supuesto, que el patrón de la respuesta de apretar la palanca haya seguido la forma de un festón invertido para las ratas 1 y 2 no es necesariamente extraño si se considera que para estas ratas la condición de entrega de agua $\mathrm{C}$ fue precedida por 30 sesiones de entrega de agua NC. Estudios previos en el área de historia conductual (Wanchisen, 1990) han mostrado que la ejecución en un programa de IF depende del programa de reforzamiento que le precede (e.g. Cole, 2001). Llama la atención que tanto las respuestas de apretar la palanca como el tiempo de la respuesta de asomarse al bebedero hayan seguido un patrón cóncavo o convexo, dependiendo de la ubicación ordinal de las condiciones de entrega de agua en ambas secuencias de exposición. Visto de esta manera, pareciera que el patrón en el interior del intervalo entre entregas de agua dependió más de factores históricos que del grado de molaridad-molecularidad de la respuesta requerida por el tipo de contingencia y, por supuesto, de la dependencia-independencia implicada en esta última en lo que a la actividad del organismo y la entrega del agua se refiere (e. g. Ribes, Carpio, Pallares \& Torres, 1986).

Tomando en cuenta los cambios en la ocurrencia y duración de las respuestas requeridas en cada condición de entrega de agua, dependiendo del tipo de contingencia implicada, los resultados del presente estudio apoyan la idea de que en un nivel funcional de análisis (Pear \& Eldridge, 1984) los condicionamientos clásico y operante son el 
derivado de un mismo proceso. El índice de ajuste calculado en cada condición de entrega de agua, sin embargo, no apoya una interpretación por el estilo. Aunque tanto para las ratas expuestas a la secuencia NC-C como para las ratas expuestas a la secuencia inversa, el índice de ajuste fue cuando menos ligeramente superior en la segunda condición de entrega de agua que en la primera, el efecto fue más robusto en el caso de la condición de entrega de agua $\mathrm{C}$ que en el caso de la entrega de agua NC. Este dato llama la atención no solo porque constituye la única diferencia observada entre el comportamiento bajo las condiciones de entrega de agua implementadas en el presente estudio, sino adicionalmente porque el efecto no corresponde con los supuestos en los que se basa en cálculo de los índices de ajuste desde el punto de vista de la psicología interconductual. De acuerdo con Serrano (2009), independientemente de la secuencia de exposición, el índice de ajuste en la condición de entrega de agua $\mathrm{NC}$ debería de ser superior al índice de ajuste en la condición de entrega de agua $\mathrm{C}$ si, como han sugerido Ribes y López (1985), cada uno de estos arreglos contingenciales constituyen las condiciones necesarias y suficientes para la evolución de comportamientos cualitativamente diferentes en virtud de implicar una complejidad funcional distinta. Nuevos estudios "molarizando" (e. g. asomarse a un orificio) y "molecularizando" (e. g. lengüetazos) las respuestas requeridas bajo las condiciones de entrega de agua $\mathrm{C}$ y $\mathrm{NC}$, respectivamente, deberán determinar si esta "anomalía” obedece al grado de molaridad-molecularidad implicado (Ribes, 2007), al tiempo que se apoya o refuta el planteamiento en torno a un mismo proceso subyacente al comportamiento bajo las condiciones de entrega de agua $\mathrm{NC}$ y $\mathrm{C}$.

En lo que a la transición entre las condiciones de reforzamiento $\mathrm{NC}$ y $\mathrm{C}$ se refiere, los efectos de tal secuencia han sido explorados principalmente en tradiciones de investigación más apegadas a los planteamientos "hullianos" que a los planteamientos "skinnerianos". Es bien conocido, por ejemplo, que la exposición a choques eléctricos inevitables interfiere con la adquisición posterior de una respuesta instrumental para escapar de tales choques (Seligman \& Maier, 1967). El efecto análogo por la entrega de estímulos apetitivos es menos conocido (Wheatley, Welker \& Miles, 1977); pero, de hecho, recientemente ha llamado la atención de algunos analistas experimentales de la conducta interesados en la adquisición de respuestas instrumentales bajo reforzamiento demorado. Snycersky, Laraway, Huitema y Poling (2004), por ejemplo, evaluaron los efectos de la entrega $\mathrm{NC}$ de agua durante una o cinco sesiones experimentales sobre la adquisición de la respuesta de apretar la palanca bajo condiciones de reforzamiento inmediato y de reforzamiento demorado. En general, bajo condiciones de reforzamiento inmediato, observaron un mayor número de respuestas a lo largo de la sesión de adquisición para el grupo de ratas expuesto a cinco sesiones de entrega de agua $\mathrm{NC}$ que para el grupo expuesto a una sola sesión, así como en comparación con el grupo para el que la fase de entrega de agua $\mathrm{NC}$ no se implementó.

En un estudio más reciente conducido por Pulido, Paz y Sosa (2008), en el que se entregó comida en lugar de agua y solo se incluyeron grupos de ratas con reforzamiento demorado, se observó que el efecto facilitador de la entrega $\mathrm{NC}$ de comida fue mayor después de 15 sesiones que después de ninguna, una o cinco sesiones experimentales. En el estudio también se observó un efecto negativo sobre la adquisición del responder después de 30 sesiones de entrega NC del estímulo y, en todos los casos, los efectos fueron interdependientes con los valores de la demora de reforzamiento. En el contexto de estos resultados, llama la atención que 30 sesiones de entrega de agua NC no hayan interferido con la adquisición de la respuesta de palanqueo para las ratas 1 y 2 del presente estudio. Al respecto, el único efecto que se puede observar en las últimas cinco sesiones de la condición de entrega de agua $\mathrm{C}$ es que la frecuencia de apretar las palancas fue mayor para las ratas expuestas inicialmente a 
dicha condición de entrega de agua, así como que independientemente de la secuencia de exposición el responder fue más elevado bajo señales auditivas que bajo señales visuales. Aunque a diferencia del estudio de Pulido et al. (2008) en el presente experimento la entrega del agua ocurrió de manera inmediata, la entrega $\mathrm{NC}$ de pellas de comida ha mostrado afectar negativamente la adquisición de la respuesta de palanqueo en ratas, bajo condiciones de reforzamiento inmediato, con tan solo 15 sesiones (e.g. Wheatley et al., 1977). Nuevos estudios deberán darse a la tarea de comparar los efectos de la entrega $\mathrm{NC}$ de estímulos apetitivos (i. e. agua y comida) bajo ambas condiciones de reforzamiento (i.e. inmediato y demorado) para aclarar este punto.

Antes de concluir, otro comentario importante en torno de la transición de la entrega de agua $\mathrm{NC} \mathrm{a}$ la $\mathrm{C}$ se relaciona con algunos estudios recientes sobre la llamada estimación temporal en animales. Al respecto, López y Menez (2012) encontraron que la exposición a la entrega $\mathrm{NC}$ de leche condensada diluida en agua facilitó el control temporal de la respuesta de apretar la palanca bajo una condición de entrega $C$, principalmente cuando los programas utilizados en ambas condiciones de reforzamiento fueron paramétricamente equivalentes. Este efecto se atribuyó a la transferencia del control temporal generado por el programa NC sobre la respuesta de asomarse al bebedero, debido ya sea a un proceso de sustitución entre las respuestas de asomarse al bebedero y apretar la palanca, a la competencia entre respuestas interinas y terminales (Staddon \& Simmelhag, 1971), o bien a una combinación de ambos procesos.

Dado que en el presente experimento también se observó un efecto de transferencia de la primera a la segunda condición de entrega de agua, pero independientemente de la secuencia de exposición y de las respuestas requeridas en cada caso - estimado a partir de los índices de ajuste - la posibilidad de un proceso de sustitución de respuestas mediado por la propiedad funcional que ambas comparten, es decir, por constituir las respuestas requeridas en cada caso y no por pertenecer a una misma clase, parece verse apoyada. En cualquier caso, dado que de acuerdo con Skinner (1938) una clase de respuesta está definida por el criterio de su reforzamiento, concebir la posibilidad de diferentes respuestas requeridas parece un recurso conceptualmente más viable, en la medida de que aunque cualquier interacción psicológica supone un requerimiento conductual para el organismo, ello no significa que bajo condiciones de entrega NC de estímulos estos ocurran por asomarse al bebedero. Más que dé una explicación ad hoc que atenta contra la coherencia de un modelo conceptual, vincular lo que en tal modelo se consideran comportamientos cualitativamente diferentes, indudablemente, será más plausible atendiendo a los parámetros por ellos compartidos, en el contexto de una aproximación conceptual más comprehensiva (Cabrer, Daza \& Ribes, 1975).

\section{Referencias}

Appel, J. B. \& Hiss, R. H. (1962). The discrimination of contingent from non-contingent reinforcement. Journal of Comparative and Physiological Psychology, 55, 37-39. doi: 10.1037/ h0044613

Brown, P. L. \& Jenkins, H. M. (1968). Auto-shaping of the pigeon's key-peck. Journal of the Experimental Analysis of Behavior, 11, 1-8. doi: 10.1901/jeab.1968.11-1

Cabrer, F., Daza, C. \& Ribes, E. (1975). Teoría de la conducta: ¿nuevos conceptos o nuevos parámetros? Revista Mexicana de Análisis de la Conducta, 1, 191-212.

Cole, M. R. (2001). The long-term effect of high- and low-rate responding histories on fixed-interval responding in rats. Journal of the Experimental Analysis of Behavior, 75, 43-54. doi: 10.1901/ jeab.2001.75-43

Estes, W. K. \& Skinner, B. F. (1941). Some quantitative properties of anxiety. Journal of Experi- 
mental Psychology, 29, 390-400. doi: 10.037/ h0062283

Holland, P. C. (2000). Trial and intertrial durations in appetitive conditioning in rats. Animal Learning \& Behavior, 28, 121-135. doi: 10.3758/ BF03200248

Jenkins, H. M. \& Moore, B. R. (1973). The form of the auto-shaped response with food or water reinforcers. Journal of the Experimental Analysis of Behavior, 20, 163-181. doi: 10.1901/ jeab.1973.20-163

Lattal, K. A. \& Maxy, G. C., (1971). Some effects of response independent reinforcers in multiple schedules. Journal of the Experimental Analysis of Behavior, 16, 225-231. doi: 10.1901/ jeab.1971.16-225

López, F. \& Menez, M. (2012). Transference effects of prior non-contingent reinforcement on the acquisition of temporal control on fixed-interval schedules. Behavioural Processes, 90, 402-407. doi: 10.1016/j.beproc.2012.04.007

Pavlov, I. P. (1927). Conditioned reflexes: An investigation of the physiological activity of the cerebral cortex. London: Oxford University Press.

Pear, J. J. \& Eldrige, G. D. (1984). The operant-respondent distinction: Future directions. Journal of the Experimental Analysis of Behavior, 42, 453-467. doi: 10.1901/jeab.1984.42-453

Pulido, M. A., Paz, M. \& Sosa, R. (2008). The effects pf behavioral history on response acquisition with delayed reinforcement: A parametric analysis. Mexican Journal of Behavior Analysis, 34, 43-56.

Ribes, E. (2007). Estados y límites del campo, medios de contacto y análisis molar del comportamiento: reflexiones teóricas. Acta Comportamentalia, 15, 229-259.

Ribes, E., Carpio, C., Pallares, A. \& Torres, J. (1986). Efectos de la reducción de la disponibilidad de reforzamiento en un programa temporal señalado. Revista Mexicana de Análisis de la Conducta, 12, 127-136.
Ribes, E. \& López, F. (1985). Teoría de la conducta: un análisis de campo y paramétrico. México: Trillas.

Schoenfeld, W. N. \& Cole, B. K. (1972). Stimulus schedules: The t- $\tau$ systems. New York: Harper and Row.

Seligman, M. E. \& Maier, S. F. (1967). Failure to escape traumatic shock. Journal of Experimental Psychology, 74, 1-9. doi: 10.1037/h0024514

Serrano, M. (2009). Complejidad e inclusividad progresivas: algunas implicaciones y evidencias empíricas en el caso de las funciones contextual, suplementaria y selectora. Revista Mexicana de Análisis de la Conducta, 35 (MONO), 161-178.

Serrano, M., Castellanos, B., Cortés-Zúñiga, A., De la Sancha-Villa, O. \& Guzmán-Díaz, G. (2011). Ajuste del comportamiento bajo programas definidos temporalmente de diferente complejidad concurrentemente disponibles. Acta Comportamentalia, 19, 137-147.

Shapiro, M. M. (1961). Salivary conditioning in dogs during fixed-interval reinforcement contingent upon lever pressing. Journal of the Experimental Analysis of Behavior, 4, 361-364. doi: 10.1901/jeab.1961.4-361

Skinner, B. F. (1938). The behavior of organisms: An experimental analysis. New York: Appleton Century Crofts.

Skinner, B. F. (1948). "Superstition" in the pigeon. Journal of Experimental Psychology, 38, 168172. doi: $10.1037 / \mathrm{h} 0055873$

Snycersky, S., Laraway, S., Huitema, B. E. \& Poling, A. (2004). The effects of behavioral history on response acquisition with immediate and delayed reinforcement. Journal of the Experimental Analysis of Behavior, 81, 51-64. doi: 10.1901/ jeab.2004.81-51

Staddon, J. E. R. \& Simmelhag, V. L. (1971). The superstition experiment: A reexamination of its implications for the principles of adaptive behavior. Psychological Review, 78, 3-43. doi: 10.1037/h0030305 
Wanchisen, B. A. (1990). Forgetting the lessons of history. The Behavior Analyst, 13, 31-37.

Weiss, S. J. \& Weissman, R. D. (1992). Generalization peck shift for autoshaped and operant key pecks. Journal of the Experimental Analysis of Behavior, 57, 127-143. doi: 10.1901/ jeab.1992.57-127

Wheatley, K. L., Welker, R. L. \& Miles, R. C (1977). Acquisition of barpressing in rats following experience with response-independent food. Animal Learning \& Behavior, 5, 236-242. doi: 10.3758/BF03209233

Williams, D. R. (1981). Biconditional behavior: Conditioning without constraint. En C. M. Locurto,
H. S. Terrace \& Gibbon, J. (Eds.), Autoshaping and conditioning theory (pp. 55-99). New York: Academic Press.

Wollin, B. R. (1968). Differences in the manner of pecking a key between pigeons reinforced with food and water. En A. C. Catania (Ed.), Contemporary research in operant behavior (p. 286). Glenview, Ill: Scott, Foresman.

Zeiler, M. D. (1968). Fixed and variable schedules of response-independent reinforcement. Journal of the Experimental Analysis of Behavior, 11, 405-414. doi: 10.1901/jeab.1968.11-405
Fecha de recepción: 8 de agosto de 2014 Fecha de aceptación: 6 de enero de 2015 
